\title{
A Numerical Method for Time Fractional Diffusion Equation Guangzhen Song ${ }^{1, a}$,Weijia Zhao ${ }^{2, b}$ and JianfeiHuang ${ }^{3, b}$ \\ ${ }^{1}$ School of Mathematics, Qingdao University, Qingdao, 266071, China \\ 2 School of Mathematics, Qingdao University, Qingdao,266071, China \\ ${ }^{3}$ School of Mathematics, Qingdao University, Qingdao, 266071, China

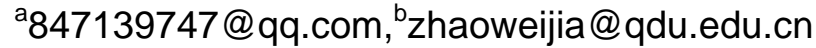

\begin{abstract}
Keywords: time fractional diffusion equation, block-by-block method, Caputo derivative.
\end{abstract}
\begin{abstract}
Use block-by-block method and classic second-order central difference approximation to discretize its temporal direction and spatial direction, respectively. This new scheme can effectively solve a class of time fractional diffusion equations with initial boundary value conditions.
\end{abstract}

\section{Introduction}

It is usually difficult to obtain the analytical solution for a fractional differential equation (FDE).So there has been a growing interest to develop numerical approaches in solving the FDE. For example, Lin and $\mathrm{Liu}^{[1]}$ developed a kind of linear multistep methods for fractional initial value problems based on Lubich's high-order approximations ${ }^{[2]}$ to fractional derivatives and integrals. In 2006, Kumar and Agrawal ${ }^{[3]}$ also utilized the equivalent Volterra integral equation in $^{[4]}$ and extended the block-by-block method proposed by Linz ${ }^{[5]}$ to some kind of FDEs. In this paper, block - by block method is extended to the solution of FDEs. The new numerical format has three advantages: spent little time in computing the starting weights and has the advantage of efficient computing time; in the direction of time and space can achieve high accuracy; the scheme is numerically stable.

\section{Block-by-block method}

Consider the following nonlinear FDE with the initial conditions:

$$
\begin{gathered}
{ }_{0}^{C} D_{t}^{\alpha} y(t)=f(t, y(t)), \quad 0 \leq t \leq T, \quad n-1<\alpha \leq n \\
y^{(k)}(0)=c_{k}, \quad k=0,1, \cdots, n-1 .
\end{gathered}
$$

In Eq. (1), ${ }^{C} D_{t}^{\alpha}$ denotes the Caputo derivative of order $\alpha$, defined by

$$
{ }_{0}^{C} D_{t}^{\alpha} y(t):=\frac{1}{\Gamma(n-\alpha)} \int_{0}^{t}(t-\tau)^{n-\alpha-1} \frac{d^{n} y(\tau)}{d \tau^{n}} d \tau
$$

If $f(t, y)$ is continuous, the initial value problem (IVP) Eq.(1-2) is equivalent to the following Volterra integral equation of the second kind[6]

where

$$
y(t)=g(t)+\frac{1}{\Gamma(\alpha)} \int_{0}^{t}(t-\tau)^{\alpha-1} f(\tau, y(\tau)) d \tau
$$

$$
g(t):=\sum_{k=0}^{n-1} c_{k} \frac{t^{k}}{k !}
$$

The specific format structure for solving initial value problems Eq. (1) and Eq. (2) is as follows. First divide the interval $[0, T]$ into $2 N$ parts with stepsize $\tau=T /(2 N)$, and set $t_{j}=j \tau,(j=0,1, \cdots, 2 N)$. The numerical solution of Eq. (3) at the point $t_{j}$ is denoted by $y_{j}$, let $g_{j}=g\left(t_{j}\right)$ and $f_{j}=f\left(t_{j}, y_{j}\right)$.

Now assume that $y_{j}(j=0,1, \ldots, 2 m)$, the approximations of $y\left(t_{j}\right)(j=0,1, \ldots, 2 m)$ are obtained, and the numerical solutions $y_{2 m+1}$ and $y_{2 m+1}$ can be obtained by the following formulas 


$$
\begin{gathered}
y_{2 m+2}=g_{2 m+2}+\sum_{k=0}^{m-1}\left[W_{k, 0}^{[2 m+2]} f_{2 k}+W_{k, 1}^{[2 m+2]} f_{2 k+1}+W_{k, 2}^{[2 m+2]} f_{2 k+2}\right] \\
+W_{m, 0}^{[2 m+2]} f_{2 m}+W_{m, 1}^{[2 m+2]} f_{2 m+1}+W_{m, 2}^{[2 m+2]} f_{2 m+2}, \\
y_{2 m+1}=g_{2 m+1}+\sum_{k=0}^{m-1}\left[W_{k, 0}^{[2 m+1]} f_{2 k}+W_{k, 1}^{[2 m+1]} f_{2 k+1}+W_{k, 2}^{[2 m+1]} f_{2 k+2}\right] \\
+W_{m, 0}^{[2 m+1]} f_{2 m}+W_{m, 1}^{[2 m+1]} f_{2 m+1}+W_{m, 2}^{[2 m+1]} f_{2 m+2},
\end{gathered}
$$

where for $i=0,1,2$,

$$
\begin{aligned}
& W_{k, i}^{[2 m+2]}:=\frac{1}{\Gamma(\alpha)} \int_{t_{2 k}}^{t_{2 k+2}}\left(t_{2 m+2}-\tau\right)^{\alpha-1} \phi_{k, i}(\tau) d \tau, \quad k=0,1, \ldots, m, \\
& W_{k, i}^{[2 m+1]}:=\frac{1}{\Gamma(\alpha)} \int_{t_{2 k}}^{t_{2 k+2}}\left(t_{2 m+1}-\tau\right)^{\alpha-1} \phi_{k, i}(\tau) d \tau, \quad k=0,1, \ldots, m-1,
\end{aligned}
$$

In Eq. (5), if $k=m, W_{m, i}^{[2 m+1]}(i=0,1,2)$ are defined as

$$
W_{m, 0}^{[2 m+1]}:=d_{2 m}+\frac{3}{8} d_{2 m+1 / 2}, \quad W_{m, 1}^{[2 m+1]}:=\frac{3}{4} d_{2 m+1 / 2}+d_{2 m+1}, \quad W_{m, 2}^{[2 m+1]}:=-\frac{1}{8} d_{2 m+1 / 2}
$$

With $\quad d_{2 m}:=\frac{1}{\Gamma(\alpha)} \int_{t_{2 m}}^{t_{2 m+1}}\left(t_{2 m+1}-\tau\right)^{\alpha-1} \psi_{m, 0}(\tau) d \tau, \quad d_{2 m+\frac{1}{2}}:=\frac{1}{\Gamma(\alpha)} \int_{t_{2 m}}^{t_{t_{m+1}}}\left(t_{2 m+1}-\tau\right)^{\alpha-1} \psi_{m, 1}(\tau) d \tau$,

$$
d_{2 m+1}:=\frac{1}{\Gamma(\alpha)} \int_{t_{2 m}}^{t_{2 m+1}}\left(t_{2 m+1}-\tau\right)^{\alpha-1} \psi_{m, 2}(\tau) d \tau,
$$

Functions $\phi_{k, i}(t)(i=0,1,2)$ are quadratic Lagrange interpolating polynomials associated with points $t_{2 k}, t_{2 k+1}$ and $t_{2 k+2}$, precisely,

$$
\phi_{k, 0}(t):=\frac{\left(t-t_{2 k+1}\right)\left(t-t_{2 k+2}\right)}{2 \tau^{2}}, \quad \phi_{k, 0}(t):=\frac{\left(t-t_{2 k}\right)\left(t-t_{2 k+2}\right)}{\tau^{2}}, \quad \phi_{k, 2}(t):=\frac{\left(t-t_{2 k}\right)\left(t-t_{2 k+1}\right)}{2 \tau^{2}},
$$

Similarly, $\quad \psi_{k, i}(t)(i=0,1,2)$ are Lagrange interpolating polynomials associated with points $t_{2 k}, t_{2 k+1 / 2}$ and $t_{2 k+1}$.From Eq.(6)-(8),it is known that $W_{k, i}^{[2 m+2]}$ and $W_{k, i}^{[2 m+1]}$ can be explicitly calculated. For simplicity, we reduce Eq. (4) and Eq.(5) to

where

$$
\begin{gathered}
y_{2 m+2}=g_{2 m+2}+\tau^{\alpha} \sum_{j=0}^{2 m} \omega_{2 m+2-j} f_{j}+\tau^{\alpha} \omega_{1} f_{2 m+1}+\tau^{\alpha} \omega_{0} f_{2 m+2}, \\
y_{2 m+1}=g_{2 m+1}+\tau^{\alpha} \sum_{j=0}^{2 m} \varpi_{2 m+2-j} f_{j}+\tau^{\alpha} \varpi_{1} f_{2 m+1}+\tau^{\alpha} \varpi_{0} f_{2 m+2},
\end{gathered}
$$

$$
\begin{aligned}
\omega_{0} & :=W_{m, 2}^{[2 m+2]} / \tau^{\alpha}, \omega_{2 \mathrm{k}+1}:=W_{m-k, 1}^{[2 m+2]} / \tau^{\alpha}, \quad k=0,1, \ldots, m ; \\
\omega_{2 \mathrm{k}} & :=W_{m-k, 2}^{[2 m+2]} / \tau^{\alpha}+W_{m-k+1,0}^{[2 m+2]} / \tau^{\alpha}, \quad k=1,2, \ldots, m ; \quad \omega_{2 m+2}:=W_{0,0}^{[2 m+2]} / \tau^{\alpha} .
\end{aligned}
$$

and $\varpi_{j}$ is defined similarly just by replacing the $W_{k, i}^{[2 m+2]}$ in the definition of $\omega_{j}$ with $W_{k, i}^{[2 m+1]}(j=0,1, \cdots, 2 m+2)$.

\section{Difference format based on block-by-block method}

Consider the following equations

$$
\begin{gathered}
{ }_{0}^{C} D_{t}^{\alpha} u(x, t)=c \frac{\partial^{2} u(x, t)}{\partial x^{2}}+f(x, t), \quad 0 \leq x \leq L, 0<t \leq T, 0<\alpha<1 . \\
u(x, 0)=\phi(x), 0 \leq x \leq L . \\
u(0, t)=u(L, t)=0, t>0 .
\end{gathered}
$$

where $c$ is a constant diffusion-wave coefficient, $f(x, t)$ is the source term.

From the section2, we know that equation (10) is equivalent to the following equation

$$
u(x, t)=\phi(x)+\frac{c}{\Gamma(\alpha)} \int_{0}^{t}(t-s)^{\alpha-1} \frac{\partial^{2} u(x, s)}{\partial x^{2}} d s+F(x, t)
$$


where $F(x, t)=J_{t}^{\alpha} f(x, t)$.

Firstly, discrete the temporal direction of Eq.(13) by the block-by-block method.

Assume that when $t=t_{n}, u^{n}(x)=u\left(x, t_{n}\right)$, where $t_{n}=n \tau, \tau=T / 2 N$.Eq.(13) can be described by the block-by-block method as follow

$u^{2 i+2}(x) \approx \phi(x)+c \tau^{\alpha} \sum_{k=0}^{2 i} \omega_{2 i+2-k} \frac{\partial^{2} u^{k}(x)}{\partial x^{2}}+c \tau^{\alpha} \omega_{1} \frac{\partial^{2} u^{2 i+1}(x)}{\partial x^{2}}+c \tau^{\alpha} \omega_{0} \frac{\partial^{2} u^{2 i+2}(x)}{\partial x^{2}}+F^{2 i+2}(x)$

$u^{2 i+1}(x) \approx \phi(x)+c \tau^{\alpha} \sum_{k=0}^{2 i} \varpi_{2 i+2-k} \frac{\partial^{2} u^{k}(x)}{\partial x^{2}}+c \tau^{\alpha} \varpi_{1} \frac{\partial^{2} u^{2 i+1}(x)}{\partial x^{2}}+c \tau^{\alpha} \varpi_{0} \frac{\partial^{2} u^{2 i+2}(x)}{\partial x^{2}}+F^{2 i+1}(x)$

where $i=0,1, \cdots N-1, \quad F^{n}(x)=F\left(x, t_{n}\right)$.

Second, discrete the spatial direction of Eq.(14) and Eq.(15).The second derivative by using second-order centered finite difference method discretize its spatial direction,

$$
\frac{\partial^{2} u^{n}\left(x_{j}\right)}{\partial x^{2}}=\frac{u^{n}\left(x_{j-1}\right)-2 u^{n}\left(x_{j}\right)+u^{n}\left(x_{j+1}\right)}{h^{2}}+O\left(h^{2}\right)
$$

here, $h=L / M, M$ is a positive integer, $x_{j}=j h$.

Put Eq. (16) to Eq.(14)and Eq.(15),omit the truncation errors, and set $u^{n}\left(x_{j}\right) \approx u_{j}^{n}$, have

$$
\begin{gathered}
u_{j}^{2 i+2} \approx \phi_{j}+\frac{c \tau^{\alpha}}{h^{2}} \sum_{k=0}^{2 i} \omega_{2 i+2-k}\left(u_{j-1}^{k}-2 u_{j}^{k}+u_{j+1}^{k}\right) \\
+\frac{c \tau^{\alpha}}{h^{2}} \omega_{1}\left(u_{j-1}^{2 i+1}-2 u_{j}^{2 i+1}+u_{j+1}^{2 i+1}\right)+\frac{c \tau^{\alpha}}{h^{2}} \omega_{0}\left(u_{j-1}^{2 i+2}-2 u_{j}^{2 i+2}+u_{j+1}^{2 i+2}\right)+F_{j}^{2 i+2} \\
u_{j}^{2 i+1} \approx \phi_{j}+\frac{c \tau^{\alpha}}{h^{2}} \sum_{k=0}^{2 i} \varpi_{2 i+2-k}\left(u_{j-1}^{k}-2 u_{j}^{k}+u_{j+1}^{k}\right)+\frac{c \tau^{\alpha}}{h^{2}} \sigma_{1}\left(u_{j-1}^{2 i+1}-2 u_{j}^{2 i+1}+u_{j+1}^{2 i+1}\right)+\frac{c \tau^{\alpha}}{h^{2}} \varpi_{0}\left(u_{j-1}^{2 i+2}-2 u_{j}^{2 i+2}+u_{j+1}^{2 i+2}\right)+F_{j}^{2 i+1}
\end{gathered}
$$

where, $\phi_{j}=\phi\left(x_{j}\right), F_{j}^{n}=F^{n}\left(x_{j}\right)=F\left(x_{j}, t_{n}\right)$.

Make $\gamma=c \tau^{\alpha} / h^{2}$, we have

$$
\begin{aligned}
& u_{j}^{2 i+2}-\gamma \omega_{1}\left(u_{j-1}^{2 i+1}-2 u_{j}^{2 i+1}+u_{j+1}^{2 i+1}\right)-\gamma \omega_{0}\left(u_{j-1}^{2 i+2}-2 u_{j}^{2 i+2}+u_{j+1}^{2 i+2}\right) \\
& =\phi_{j}+\gamma \sum_{k=0}^{2 i} \omega_{2 i+2-k}\left(u_{j-1}^{k}-2 u_{j}^{k}+u_{j+1}^{k}\right)+F_{j}^{2 i+2} \\
& u_{j}^{2 i+1}-\gamma \varpi_{1}\left(u_{j-1}^{2 i+1}-2 u_{j}^{2 i+1}+u_{j+1}^{2 i+1}\right)-\gamma \varpi_{0}\left(u_{j-1}^{2 i+2}-2 u_{j}^{2 i+2}+u_{j+1}^{2 i+2}\right) \\
& =\phi_{j}+\gamma \sum_{k=0}^{2 i} \varpi_{2 i+2-k}\left(u_{j-1}^{k}-2 u_{j}^{k}+u_{j+1}^{k}\right)+F_{j}^{2 i+1}
\end{aligned}
$$

Introduction of symbols: $A=I-\gamma \omega_{0} B, \widetilde{A}=I-\gamma \varpi_{0} B$, where $I$ is identity matrix with $(M-1) \times(M-1)$,

$$
B=\left(\begin{array}{cccccc}
-2 & 1 & & & & \\
1 & -2 & 1 & & & \\
& \cdots & \cdots & \cdots & & \\
& & & 1 & -2 & 1 \\
& & & & 1 & -2
\end{array}\right)
$$

Then we have $A\left(\begin{array}{c}u_{1}^{2 i+2} \\ u_{2}^{2 i+2} \\ \ldots \\ u_{M-1}^{2 i+2}\end{array}\right)-\gamma \omega_{1} B\left(\begin{array}{c}u_{1}^{2 i+1} \\ u_{2}^{2 i+1} \\ \ldots \\ u_{M-1}^{2 i+1}\end{array}\right)=\left(\begin{array}{c}\phi_{1} \\ \phi_{2} \\ \ldots \\ \phi_{M-1}\end{array}\right)+\left(\begin{array}{c}F_{1}^{2 i+2} \\ F_{2}^{2 i+2} \\ \ldots \\ F_{M-1}^{2 i+2}\end{array}\right)+\gamma \sum_{k=0}^{2 i} \omega_{2 i+2-k} B\left(\begin{array}{c}u_{1}^{k} \\ u_{2}^{k} \\ \ldots \\ u_{M-1}^{k}\end{array}\right)$ 


$$
-\gamma \varpi_{0} B\left(\begin{array}{c}
u_{1}^{2 i+2} \\
u_{2}^{2 i+2} \\
\ldots \\
u_{M-2}^{2 i+2}
\end{array}\right)+\tilde{A}\left(\begin{array}{c}
u_{1}^{2 i+1} \\
u_{2}^{2 i+1} \\
\ldots \\
u_{M-1}^{2 i+1}
\end{array}\right)=\left(\begin{array}{c}
\phi_{1} \\
\phi_{2} \\
\ldots \\
\phi_{M-1}
\end{array}\right)+\left(\begin{array}{c}
F_{1}^{2 i+1} \\
F_{2}^{2 i+1} \\
\ldots \\
F_{M-1}^{2 i+1}
\end{array}\right)+\gamma \sum_{k=0}^{2 i} \varpi_{2 i+2-k} B\left(\begin{array}{c}
u_{1}^{k} \\
u_{2}^{k} \\
\ldots \\
u_{M-1}^{k}
\end{array}\right)
$$

Comprehensive above two mathematical expressions, the algorithm can be expressed as the

following matrix form $\left(\begin{array}{cc}-\gamma \omega_{1} B & A \\ \tilde{A} & -\gamma \varpi_{0} B\end{array}\right)\left(\begin{array}{c}u_{1}^{2 i+1} \\ \ldots \\ u_{M-1}^{2 i+1} \\ u_{1}^{2 i+2} \\ \ldots \\ u_{M-1}^{2 i+2}\end{array}\right)=\left(\begin{array}{c}\phi_{1} \\ \ldots \\ \phi_{M-1} \\ \phi_{1} \\ \ldots \\ \phi_{M-1}\end{array}\right)+\left(\begin{array}{c}F_{1}^{2 i+1} \\ \ldots \\ F_{M-1}^{2 i+1} \\ F_{1}^{2 i+2} \\ \ldots \\ F_{M-1}^{2 i+2}\end{array}\right)+\gamma \sum_{k=0}^{2 i}\left(\begin{array}{c}\omega_{2 i+2-k} B \\ \varpi_{2 i+2-k} B\end{array}\right)\left(\begin{array}{c}u_{1}^{k} \\ u_{2}^{k} \\ \ldots \\ u_{M-1}^{k}\end{array}\right)$

\section{Numerical experiment}

In order to verify the analysis of schemes, consider the following equation

$$
\begin{gathered}
{ }_{0}^{C} D_{t}^{\alpha} u(x, t)=\frac{\partial^{2} u(x, t)}{\partial x^{2}}+f(x, t), 0<x \leq 1,0<\alpha<1 \\
u(x, 0)=0, u_{t}(x, 0)=0,0 \leq x \leq 1 ; u(0, t)=u(1, t)=0,0<t \leq 1
\end{gathered}
$$

The exact solution of this fractional differential equation is $u(x, t)=0.5 t^{3+\alpha} \sin (\pi x)$.

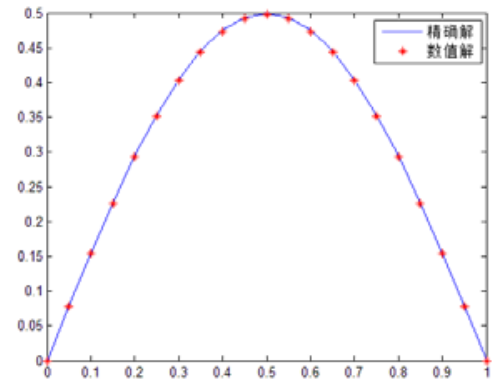

Fig. 1: The comparison of numerical solution and exact solution for $\alpha=0.9, h=\tau=0.05$

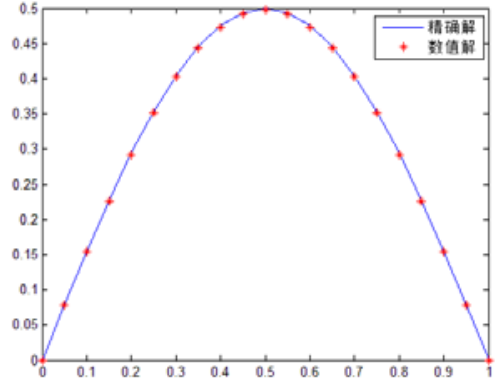

Fig. 2: The comparison of numerical solution and exact solution for $\alpha=0.8, h=0.05$ and $\tau=0.0125$

The comparisons of numerical solution and exact solution for $\alpha=0.8$ are shown in fig.1and fig.2. For this case, take stepsize $h=\tau=0.05$ and $h=0.05, \tau=0.0125$.It can be seen that numerical results are in excellent agreement with the exact solution. From table1 and table 2, as the stepsize decreasing, the error is reduced. Thus the numerical results are consistent with the theoretical analysis in Section 3.

Table 1: The errors for different stepsize $h=\tau \quad$ Table 2: The errors for different stepsize $h$ and $\alpha=0.5, \alpha=0.8$ and

\begin{tabular}{l|l|l}
\hline \multirow{2}{*}{$h=\tau$} & $\alpha=0.5$ & $\alpha=0.8$ \\
\cline { 2 - 3 } & $\sqrt{\Sigma\left(h * E^{2}\right)}$ & $\sqrt{\Sigma\left(h * E^{2}\right)}$ \\
\hline $1 / 10$ & $6.6000 \times 10^{-3}$ & $4.9000 \times 10^{-3}$ \\
\hline $1 / 20$ & $4.7000 \times 10^{-3}$ & $1.8000 \times 10^{-3}$ \\
\hline $1 / 40$ & $2.4000 \times 10^{-3}$ & $5.1578 \times 10^{-4}$ \\
\hline $1 / 80$ & $1.1000 \times 10^{-3}$ & $1.3968 \times 10^{-4}$ \\
\hline $1 / 160$ & $4.5362 \times 10^{-4}$ & $3.8280 \times 10^{-5}$ \\
\hline
\end{tabular}
$\alpha=0.5, \alpha=0.8$

\begin{tabular}{c|l|l}
\hline \multirow{2}{*}{$h$} & $\alpha=0.5$ & $\alpha=0.8$ \\
\cline { 2 - 3 } & $\sqrt{\Sigma\left(h * E^{2}\right)}$ & $\sqrt{\Sigma\left(h * E^{2}\right)}$ \\
\hline $1 / 10$ & $8.5000 \times 10^{-3}$ & $2.8000 \times 10^{-3}$ \\
\hline $1 / 20$ & $4.7000 \times 10^{-3}$ & $1.8000 \times 10^{-3}$ \\
\hline $1 / 40$ & $2.4000 \times 10^{-3}$ & $9.5835 \times 10^{-4}$ \\
\hline $1 / 80$ & $1.2000 \times 10^{-3}$ & $4.8561 \times 10^{-4}$ \\
\hline $1 / 160$ & $6.0864 \times 10^{-4}$ & $2.4361 \times 10^{-4}$ \\
\hline
\end{tabular}




\section{Concluding remarks}

In this paper we have constructed one finite difference scheme for time fractional diffusion-wave equation based on its equivalent partial integro- differential equation. The numerical examples have verified the theoretical results. It is an effective and convergent numerical scheme in solving a variety of FDEs.

\section{References}

[1] R.Linz and F.Liu, Fractional high order methods for the nonlinear fractional ordinary differential equation, Nonlinear Dynam. 66 (2007) ,pp.856-869.

[2] Ch.Lubich, Discretized fractional calculus, SIAM J, Math. Anal. vol .17,no.3,(1986), pp.704-719.

[3] P.Kumar and O.P.Agrawal, An approximate method for numerical solution of fractional differential equations, Singal Process. 86(2006), pp.2602-2610.

[4] K.Diethelm, N.J.Ford, and A.D.Freed, A predictor-corrector approach for the numerical solution of fractional differential equations, Nonlinear Dynam.29(2002),pp.3-22.

[5] R.Linz, An method for nonlinear solving Volterra integral equations of the second kind, Math.Comput.vol.23,no.107,(1969),pp.595-599.

[6] K.Diethelm, The Analysis of Fractional Differential Equations. Springer, Berlin, 2010 